Systematic Review

\title{
Quadriceps tendon has a lower re-rupture rate than hamstring tendon autograft for anterior cruciate ligament reconstruction - A meta-analysis
}

\author{
Eoghan T. Hurley, MB BCh MCh PhD ", Edward S. Mojica, BS, Ajay C. Kanakamedala, MD, \\ Robert J. Meislin, MD, Eric J. Strauss, MD, Kirk A. Campbell, MD, Michael J. Alaia, MD \\ New York University Langone Health, Department of Orthopaedic Surgery, New York, NY, USA
}

\section{A R T I C L E I N F O}

\section{Keywords:}

Quadriceps tendon

Autograft

Systematic review

Anterior cruciate ligament

Hamstring tendon

\begin{abstract}
A B S T R A C T
Importance: There have been several recent systematic reviews of quadriceps tendon autografts (QT), which have not shown any significant difference in outcomes between QT and hamstring tendon autograft (HS) for ACL reconstruction (ACLR). However, several recent comparative studies have been published comparing QT to HS for ACLR.

Aim: The purpose of this study is to perform a systematic review and meta-analysis of the studies comparing QT to HS for ACLR.

Evidence review: Two independent reviewers performed the literature search based on the PRISMA guidelines, with a senior author arbitrating discrepancies. Cohort studies comparing QT with HS were included.

Findings: There were 15 studies comparing 611 patients with QT to 543 patients with HS, with a mean of 27.4 months follow-up. QT resulted in a significantly lower rate of graft re-rupture $(2.5 \% \mathrm{vs} 8.7 \%, \mathrm{p}=0.01)$, and donor site morbidity $(17.6 \%$ vs $26.2 \%, \mathrm{p}=0.02)$. There was a significant difference in favour of QT for the positive pivot shift test (Grade I/II: $15.8 \%$ vs $23.0 \%$, p = 0.02), but not in the rate of the positive Lachman test (Grade I/II: $18.3 \%$ vs $26.7 \%, \mathrm{p}=0.16$ ). Additionally, there was no difference in the side to side difference in knee stability (1.8 mm vs $2.0 \mathrm{~mm}, \mathrm{p}=0.48$ ). Functionally, both grafts had similar functional outcomes in terms of the IKDC score (88.0 vs $87.9, \mathrm{p}=0.69$ ), and Lysholm score (89.3 vs $87.6, \mathrm{p}=0.15$ ).

Conclusions and relevance: Our study showed that QT has a lower re-rupture rate than HS in ACLR, with lower donor site morbidity. QT appeared to be slightly better for residual pivot shift, but there was no difference in patient-reported outcomes.

Level of evidence: III.
\end{abstract}

What is already known

- Traditionally, surgeons have debated whether bone-patella tendon-bone autografts (BPTB) and hamstring tendon autografts (HS) should be the graft of choice in young patients.

- However, in recent years, there has been increased interest in the study of quadriceps tendon autografts (QT).

- There have been several recent systematic reviews of QT, which have not shown any significant difference in outcomes between QT and BTB or between QT and HS for ACLR.

\footnotetext{
* Corresponding author.

E-mail address: eoghan.hurley@nyulangone.org (E.T. Hurley).
}

What are the new findings

- Our systematic review and meta-analysis showed that QT has a lower re-rupture rate than HS in ACLR, with lower donor site morbidity.

- QT appeared to be slightly better for residual knee laxity, but there was no difference in patient-reported outcomes.

\section{Introduction}

An anterior cruciate ligament (ACL) rupture is a common injury, with an estimated 100,000-200,000 ACL ruptures every year in the United States alone. ACL reconstruction (ACLR), when indicated, remains the 
standard of care to limit instability and prevent further cartilage and/or meniscal pathology. Despite numerous randomized controlled trials, the choice of graft used is multifactorial and largely dependent on patient factors, as well as surgeon preference. Graft selection can be divided into three broad categories; autograft, allograft, and synthetic graft, with autografts having the lowest re-rupture rate and synthetic grafts largely out of favour. Traditionally, surgeons have debated whether bone-patella tendon-bone autografts (BPTB) and hamstring tendon autografts (HS) should be the graft of choice in young patients. However, in recent years there has been increased interest in the study of quadriceps tendon autografts (QT).

QT graft use for ACLR was first described by Marshall et al., in 1979, and the use of QT fell out of favour due to early studies showing poor biomechanical properties and unsatisfactory clinical outcomes. [12]. Many of these studies utilized a quadriceps tendon "substitution" graft consisting of a quadriceps tendon-prepatellar retinaculum-patellar tendon construct; however, an interest in QT experienced a resurgence in the 1990s when techniques describing a free quadriceps tendon graft exhibited improved biomechanical properties [3] and clinical outcomes. [4]. With modern QT harvesting techniques, there has been predicted a rise in the use of QT [5,6], with many strongly advocating for its use. [7].

There have been five recent systematic reviews on QT, which have not shown any significant difference in outcomes between QT and BTB or between QT and HS for ACLR. [8-12]. Since these prior systematic reviews, several new studies have been published comparing QT and HS, including several randomized control trials, warranting an updated meta-analysis. Therefore, the purpose of this study is to perform a systematic review and meta-analysis of the studies comparing QT to HS for ACLR. Our primary hypothesis was that QT would result in a lower failure rate than HS for ACLR.

\section{Methods}

\section{Search strategy}

For identifying clinical studies comparing QT to HS in ACLR, three databases (MEDLINE, EMBASE and The Cochrane Library) were searched in April 2020. The following search algorithm was used: (quadriceps autograft OR quadriceps tendon autograft) AND (hamstring tendon or hamstring tendon autograft) AND (ACL OR anterior cruciate ligament OR ACL reconstruction OR anterior cruciate ligament reconstruction). No constraints were placed on the publication date. Ethical approval was not required as this is a systematic review of the literature.

\section{Study selection}

Two reviewers performed the literature search based on the Preferred Reporting Items for Systematic Reviews and Meta-Analyses (PRISMA) guidelines and screened the search results, with a third author arbitrating in the event of a disagreement. The title and abstract were screened for all search results, and potentially eligible studies received a full-text review. In addition, the reference lists of all included studies and all literature reviews found in the search results were manually screened for additional articles that met the inclusion criteria.

\section{Eligibility criteria}

The inclusion criteria were: (1) clinical studies comparing QT to HS in ACLR, (2) published in a peer reviewed journal, (3) English language studies, (4) full text of studies available. The exclusion criteria were: (1) registry studies, (2) case series, (3) review studies, (4) cadaver studies, (5) biomechanical studies, (6) abstract only.

\section{Data extraction/analysis}

Relevant data were extracted from the included studies by two independent reviewers using a predetermined datasheet. When required information was not available in the text, the authors of the original studies were contacted. The methodological quality of the evidence (MQOE) was assessed using the Newcastle-Ottawa scale, a 9-point scale where studies with $7-9,5-6,4$, and $0-3$ points were identified as very good, good, satisfactory, and unsatisfactory, respectively. Outcomes analyzed were (1) ACL re-rupture, (2) donor site morbidity, (3) knee stability; Lachman test (Grade I/II), pivot shift test (Grade I/II), anterior laxity (side to side difference), and (4) functional outcomes; International Knee Documentation Committee (IKDC) score, and Lysholm score.

\section{Statistics}

All statistical analysis was performed using Review Manager ((RevMan) [Macintosh]. Version 5.3. Copenhagen: The Nordic Cochrane Centre, The Cochrane Collaboration, 2014.). Heterogeneity between studies was quantified using the $\mathrm{I}^{2}$ statistic. Random-effects models were used when there was heterogeneity $>50 \%$, and fixed-effects models were used otherwise. Results were presented in terms of risk ratio (RR) for dichotomous outcomes and mean difference (MD) for continuous outcomes, with a $95 \%$ confidence interval $(95 \% \mathrm{CI})$. A p-value of $<0.5$ was considered to be statistically significant.

\section{Results}

\section{Literature search}

The initial literature search resulted in 361 total studies. Once duplicates were removed and the articles were screened for inclusion and exclusion criteria, 29 studies were included, and full texts were assessed for eligibility. Fifteen clinical trials with 1154 patients were included in this review (Fig. 1).

\section{Study characteristics and patient demographics}

There were 15 studies [13-27] (Level of evidence I: 3; II: 2; LOE III: 10) comparing 611 patients with QT to 543 patients with HS, with a mean follow-up time of 27.4 months. The mean age was 27.9 , and $71.1 \%$ were male. The study characteristics and patient demographics are reported in Table 1.

\section{Clinical outcomes}

\section{ACL Re-Rupture (Fig. 2)}

ACL re-rupture was reported in nine studies, with 314 patients treated with QT and 323 patients with HS. Overall, 2.5\% of QT patients had graft rupture, and of those with $\mathrm{HS}, 8.7 \%$ had graft rupture. There was a statistically significant difference in favour of QT (RR; $0.36,95 \%$ CI, 0.16 to $0.79, \mathrm{I}^{2}=0 \%, \mathrm{p}=0.01$ ).

\section{Donor site morbidity (Fig. 3)}

Donor site morbidity was reported in seven studies, with 239 treated with QT and 229 patients with HS. Overall, $17.6 \%$ of QT patients had donor site morbidity, and of those with HS, $26.2 \%$ had donor site morbidity. There was a statistically significant difference in favour of QT (RR; 0.67, 95\% CI, 0.48 to $0.95, \mathrm{I}^{2}=4 \%, \mathrm{p}=0.02$ ).

\section{Pivot shift test (Fig. 4)}

A positive pivot shift test (Grade I/II) was reported in seven studies, with 386 patients treated with QT and 304 patients with HS. Overall, $15.8 \%$ of QT patients had a positive pivot shift test, and of those with HS, 


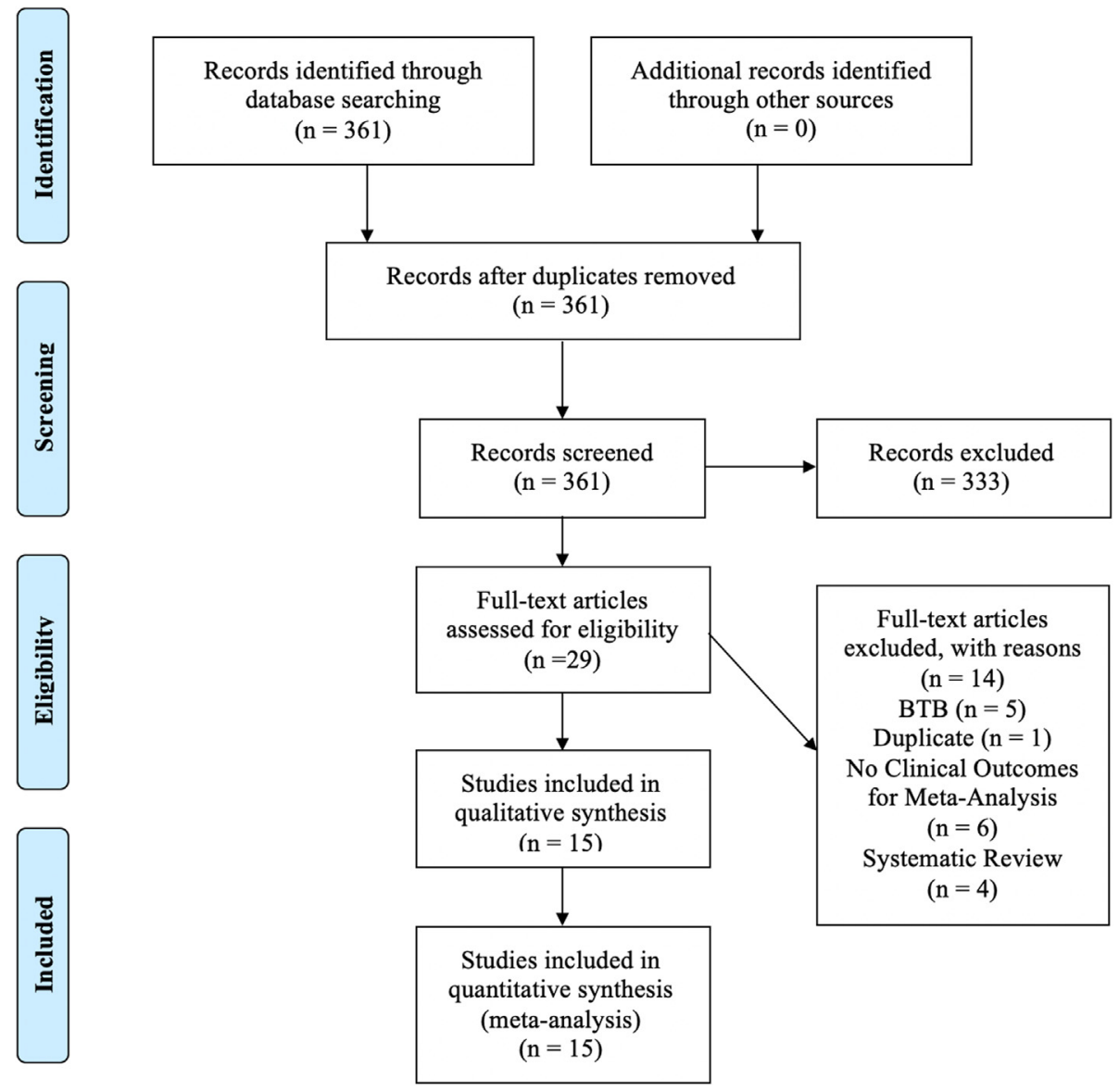

Fig. 1. PRISMA study selection flow diagram.

Table 1

Study characteristics and patient demographics.

\begin{tabular}{|c|c|c|c|c|c|c|}
\hline Author & LOE & MQOE & N (QT/HS) & Age (yrs.) & $\mathrm{M} / \mathrm{F}$ & Follow-up (mo.) \\
\hline Akoto et al., 2019 [14] & III & 8 & $82(41 / 41)$ & $28.5 \pm 10$ & $64 / 18$ & $13.9(12+)$ \\
\hline Barie et al., 2019 [15] & III & 7 & $78(41 / 37)$ & $31 \pm 10$ & $57 / 22$ & $52.3(40-66)$ \\
\hline Cavaignac et al., 2017 [16] & III & 8 & $83(44 / 39)$ & $31.5 \pm 8.5$ & $45 / 38$ & $43(36+)$ \\
\hline Haner et al., 2016 [17] & II & 8 & $51(26 / 25)$ & $35.8 \pm 11.8$ & $35 / 16$ & $24+$ \\
\hline Hart et al., 2010 [18] & III & 4 & $40(20 / 20)$ & $27 \pm 7$ & $26 / 14$ & $12+$ \\
\hline Kim et al., 2014 [20] & II & 6 & $207(142 / 65)$ & $\mathrm{N} / \mathrm{R}$ & & $24+$ \\
\hline Lee et al., 2016 [19] & III & 8 & $96(48 / 48)$ & $30.5 \pm 10.2$ & $88 / 8$ & $34.9(24-61)$ \\
\hline Lind et al., 2019 [21] & I & 9 & $99(50 / 49)$ & $27.2 \pm 6.3$ & $54 / 45$ & $24+$ \\
\hline Martin-Alguacil et al., 2018 [22] & I & 8 & $51(26 / 25)$ & $18.9 \pm 3.6$ & $39 / 9$ & $12+$ \\
\hline Mouarbes et al., 2020 [13] & III & 7 & $60(30 / 30)$ & $26.5 \pm 8.6$ & $47 / 13$ & $12+$ \\
\hline Pennock et al., 2019 [23] & III & 9 & $83(27 / 56)$ & $14.8 \pm 1.4$ & $57 / 26$ & $33.6(24+)$ \\
\hline Runer et al., 2018 [24] & III & 9 & $80(40 / 40)$ & $34.5 \pm 11.0$ & $46 / 34$ & $24+$ \\
\hline Sofu et al., 2013 [25] & III & 7 & $44(23 / 21)$ & 27.7 & $42 / 2$ & $37.6(8-70)$ \\
\hline Todor et al., 2019 [26] & III & 8 & $72(39 / 33)$ & $29.7 \pm 7.8$ & $49 / 23$ & $34.1(24+)$ \\
\hline Vilchez-Cavazos et al., 2020 [27] & I & 8 & $28(14 / 14)$ & 23 & $23 / 5$ & $12+$ \\
\hline
\end{tabular}

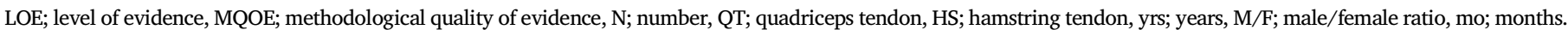

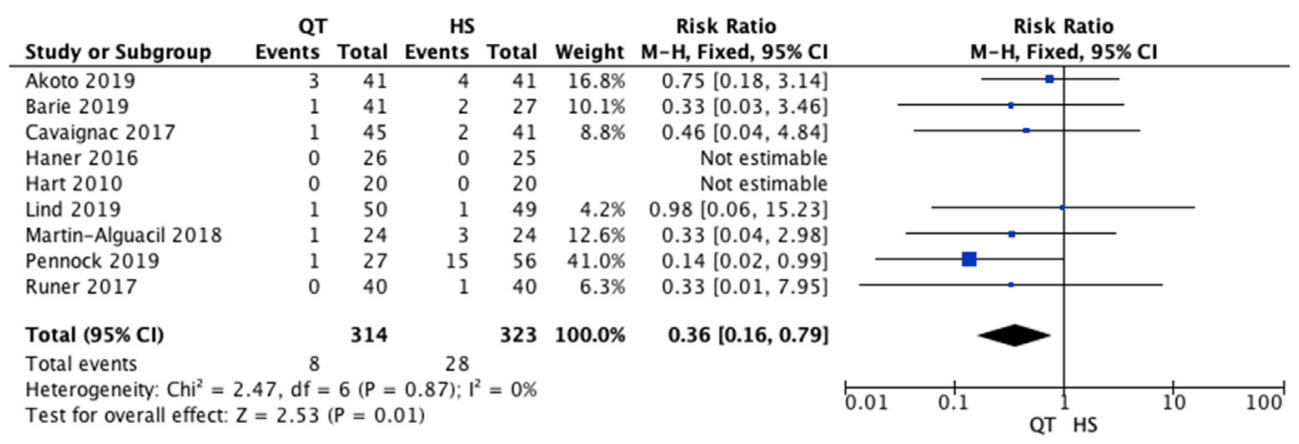

Fig. 2. Forest plot of ACL re-ruptures. 


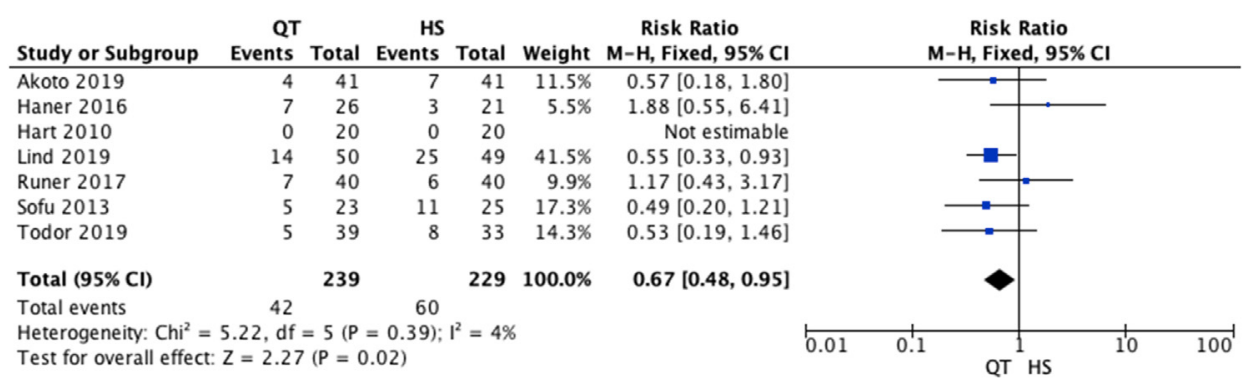

Fig. 3. Forest plot of donor site morbidity.

\begin{tabular}{|c|c|c|c|c|c|c|c|c|c|}
\hline \multirow{2}{*}{ Study or Subgroup } & $\begin{array}{r}\text { QT } \\
\text { Events }\end{array}$ & Total & \multirow{2}{*}{$\begin{array}{r}\begin{array}{r}\text { HS } \\
\text { Events }\end{array} \\
2\end{array}$} & \multirow{2}{*}{$\begin{array}{r}\text { Total } \\
41\end{array}$} & \multirow{2}{*}{$\begin{array}{r}\text { Weight } \\
2.7 \%\end{array}$} & \multirow{2}{*}{$\begin{array}{c}\begin{array}{c}\text { Risk Ratio } \\
\text { M-H, Fixed, 95\% Cl }\end{array} \\
3.00[0.64,14.00]\end{array}$} & & \multicolumn{2}{|c|}{$\begin{array}{c}\text { Risk Ratio } \\
\text { M-H, Fixed, 95\% CI }\end{array}$} \\
\hline & 6 & 41 & & & & & & & \\
\hline Barie 2019 & 6 & 41 & 15 & 37 & $20.9 \%$ & $0.36[0.16,0.83]$ & & $=$ & \\
\hline Cavaignac 2017 & 4 & 44 & 14 & 44 & $18.6 \%$ & $0.29[0.10,0.80]$ & & & \\
\hline Hart 2010 & 0 & 20 & 0 & 20 & & Not estimable & & & \\
\hline Kim 2013 & 25 & 142 & 12 & 65 & $21.9 \%$ & $0.95[0.51,1.78]$ & & $\longrightarrow$ & - \\
\hline Lee 2016 & 13 & 48 & 16 & 48 & $21.2 \%$ & $0.81[0.44,1.50]$ & & $\rightarrow$ & \\
\hline Lind 2019 & 7 & 50 & 11 & 49 & $14.7 \%$ & $0.62[0.26,1.48]$ & & & \\
\hline Total $(95 \% \mathrm{CI})$ & & 386 & & 304 & $100.0 \%$ & $0.68[0.50,0.93]$ & & & \\
\hline Total events & 61 & & 70 & & & & & & \\
\hline $\begin{array}{l}\text { Heterogeneity: } \mathrm{Chi}^{2}= \\
\text { Test for overall effect }\end{array}$ & $\begin{array}{l}9.99, \mathrm{df} \\
Z=2.38\end{array}$ & $\begin{array}{l}=5(P \\
=(P=0\end{array}$ & $\begin{array}{l}=0.08 \text { ); } \\
(.02)\end{array}$ & $I^{2}=50$ & & & 0.01 & 0.1 & I HS \\
\hline
\end{tabular}

Fig. 4. Forest plot of positive pivot shift tests.

\begin{tabular}{|c|c|c|c|c|c|c|c|c|c|c|}
\hline Study or Subgroup & $\begin{array}{r}\text { QT } \\
\text { Events }\end{array}$ & Total & $\begin{array}{r}\text { HS } \\
\text { Events }\end{array}$ & Total & Weight & $\begin{array}{c}\text { Risk Ratio } \\
\text { M-H, Random, } 95 \% \mathrm{CI}\end{array}$ & \multicolumn{4}{|c|}{$\begin{array}{c}\text { Risk Ratio } \\
\text { M-H, Random, } 95 \% \text { CI }\end{array}$} \\
\hline Akoto 2019 & 8 & 41 & 6 & 41 & $16.6 \%$ & $1.33[0.51,3.50]$ & & & & \\
\hline Cavaignac 2017 & 4 & 44 & 21 & 39 & $16.3 \%$ & $0.17[0.06,0.45]$ & & 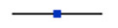 & & \\
\hline Hart 2010 & 0 & 20 & 0 & 20 & & Not estimable & & & & \\
\hline Kim 2013 & 30 & 142 & 16 & 65 & $25.0 \%$ & $0.86[0.50,1.46]$ & & & & \\
\hline Lee 2016 & 14 & 48 & 16 & 48 & $23.7 \%$ & $0.88[0.48,1.59]$ & & & & \\
\hline Lind 2019 & 7 & 50 & 11 & 49 & $18.4 \%$ & $0.62[0.26,1.48]$ & & & & \\
\hline Total $(95 \% \mathrm{Cl})$ & & 345 & & 262 & $100.0 \%$ & $0.67[0.38,1.18]$ & & & & \\
\hline Total events & 63 & & 70 & & & & & & & \\
\hline $\begin{array}{l}\text { Heterogeneity: Taü } \\
\text { Test for overall effec }\end{array}$ & $\begin{array}{l}0.26 ; \mathrm{Cr} \\
\mathrm{Z}=1.39\end{array}$ & $\begin{array}{l}i^{2}=1 \\
(P=0\end{array}$ & $\begin{array}{l}1.24, \mathrm{df}= \\
0.16)\end{array}$ & $=4(P=$ & $=0.02) ; I^{2}$ & $=64 \%$ & 0.01 & 0.1 & $T_{\mathrm{HS}}$ & 100 \\
\hline
\end{tabular}

Fig. 5. Forest plot of positive Lachman tests.

$23.0 \%$ had a positive pivot shift test. There was a statistically significant difference in favour of QT (RR; $0.68,95 \%$ CI, 0.50 to $0.93, \mathrm{I}^{2}=50 \%$, $\mathrm{p}=0.02$ ).

\section{Lachman test (Fig. 5)}

A positive Lachman test (Grade I/II) was reported in six studies, with 345 patients treated with QT and 262 patients with HS. Overall, $18.3 \%$ of QT patients had a positive Lachman test, and with HS, $26.7 \%$ had a positive Lachman test. There was no statistically significant difference (RR; $0.67,95 \%$ CI, 0.38 to $1.18, \mathrm{I}^{2}=64 \%$, $\mathrm{p}=0.16)$.

Anterior laxity (Side-Side Difference) (Fig. 6)

Anterior laxity was reported in eight studies, with 293 patients treated with QT and 280 patients with HS. The mean anterior laxity was
$1.8 \mathrm{~mm}$ with QT, and the mean anterior laxity was $2.0 \mathrm{~mm}$ with $\mathrm{HS}$. There was no statistically significant difference (RR; $-0.23,95 \% \mathrm{CI}$, -0.87 to $\left.0.41, \mathrm{I}^{2}=95 \%, \mathrm{p}=0.48\right)$.

\section{International Knee Documentation Committee score (Fig. 7)}

The IKDC score was reported in six studies, with 339 patients treated with QT and 256 patients with HS. The mean IKDC score was 88.0 with QT, and the mean IKDC Score was 87.9 with HS. There was no statistically significant difference (MD; $0.20,95 \% \mathrm{CI},-0.76$ to $1.15, \mathrm{I}^{2}=0 \%$, $\mathrm{p}=0.69$ ).

\section{Lysholm score (Fig. 8)}

Lysholm score was reported in seven studies, with 365 patients treated with QT and 279 patients with HS. The mean Lysholm score was 89.3 with QT, and the mean Lysholm score was 87.6 with HS. There was

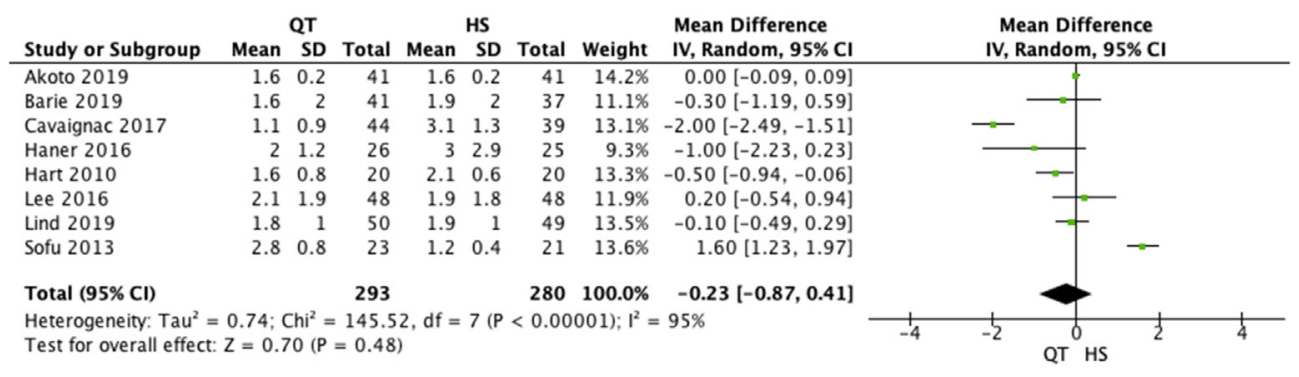

Fig. 6. Forest plot of anterior laxity (side-side difference). 


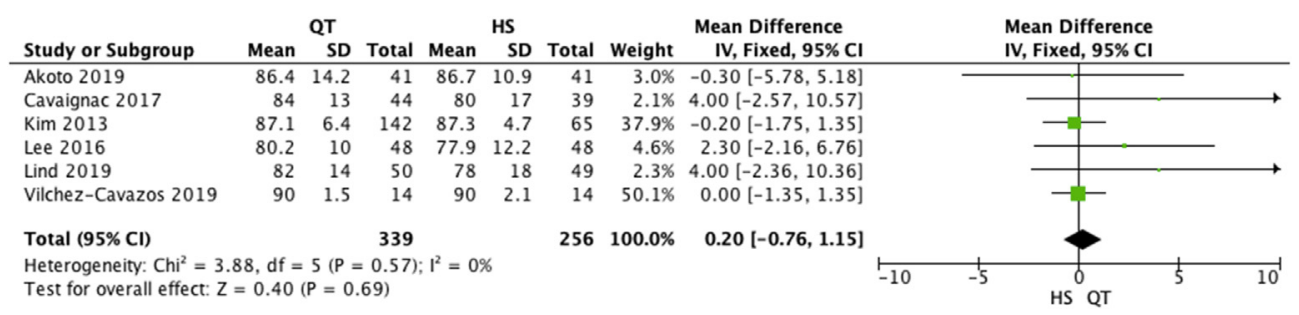

Fig. 7. Forest plot of the IKDC score.

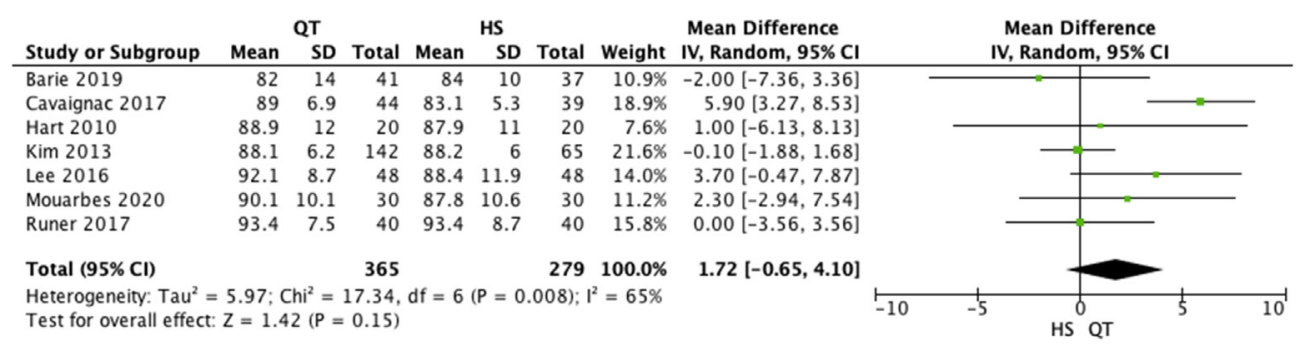

Fig. 8. Forest plot of the Lysholm score.

no statistically significant difference (MD; $1.72,95 \% \mathrm{CI},-0.65$ to 4.10 , $\left.\mathrm{I}^{2}=65 \%, \mathrm{p}=0.15\right)$.

\section{Discussion}

The most important finding of this study was that the use of QT in ACLR appears to, at short term follow up, have a significantly lower rate of graft re-rupture than HS, as well as a significantly lower incidence of donor site morbidity, thus supporting the hypothesis under study. QT appeared to be slightly better for residual knee laxity, but there was no difference in patient-reported outcomes. These findings are important for clinical practice as they suggest that QT is an acceptable graft choice in ACLR and may have some potential benefits over HS.

This is an important novel finding given that prior systematic reviews and meta-analyses of QT versus HS [8-12] have not found a difference in graft re-rupture rate or were unable to perform a quantitative meta-analysis due to fewer available studies at the time of their publication. Mouarbes et al. [11] performed a systematic review and meta-analysis that included a total of 357 patients across five studies comparing QT and HS, and in contrast to the results here, found no difference in graft re-rupture rate or donor site pain. While this review included fewer studies, it is also worth noting that they only examined donor site pain and no other aspects of donor site morbidity. Although Neyland et al. recently published a meta-analysis in which they similarly found a significantly lower rate of graft re-rupture with QT compared to HS, it is possible that there is considerable confounding from their inclusion of case series and single cohort studies. [12]. In contrast to the findings here, they also found significantly less post-operative pivot shift laxity with QT compared to HS. It is important to note that in contrast to Neyland et al., the present review only included comparative studies that directly contrast the two modalities and thus distinguishes itself from previous reviews comparing outcomes in studies not comparing hamstring and quadriceps tendon grafts.

With the inclusion of the aforementioned more recent studies and increased power in the statistical analysis, QT was found to have a lower graft re-rupture rate than HS. There are several possible explanations for the lower re-rupture rate, including graft thickness, variation in HS configuration, including the number of strands, and methods of graft fixation. A majority of the studies included in this review performed a QT harvest technique that utilized a patellar bone block. It is possible that the addition of bone-to-bone healing, which has been shown to be superior to tendon-to-bone healing $[28,29]$, could lead to reduced graft re-rupture rates with QT. Sasaki et al. performed a cadaveric study in which they compared a QT autograft, including a patellar bone block, to a quadrupled semitendinosus gracilis autograft. [30]. They found that both restored anterior tibial translation to within $2.5 \mathrm{~mm}$ of the native ACL and found no differences in pivot shift laxity or in situ forces seen within the graft, but they did not assess ultimate load to failure.

Harris et al. compared cadaveric QT to BTB grafts and found that QT grafts had on average 1.8 times the cross-sectional area of BTB grafts and an ultimate load to failure 1.36 times of BTB grafts; however, these did not reach statistical significance. ${ }^{3}$ Staubli et al. and Shani et al. performed similar studies in which they also found greater cross-sectional area and ultimate tensile load with QT compared to BTB, and their findings did reach statistical significance. [31,32]. Krystsis et al. [33] investigated the relationship between tensile load and the interplay of forces exerting upon the graft with a study utilizing Biodex technology. In it, they identified low hamstring strength relative to the quadriceps as a possible risk factor for rupture, quantified by isometric strength testing. The hamstring acts as a natural agonist of the ACL, resisting anterior tibial displacement caused by the action of the quadriceps and thus dispelling some of the forces exerted. Therefore, harvesting of the hamstring and its resulting weakening may be a risk factor, and harvesting of the quadriceps tendon may contribute to the opposite. While none of these studies directly compared the biomechanical properties of QT to HS, reported mean cross-sectional areas for QT have ranged from 61.9 to $91.3 \mathrm{~mm}^{2}$ [31,32] versus $35.3-57 \mathrm{~mm}^{2}$ [34-36] for quadruple stranded hamstring tendon.

QT was also found to have significantly less donor site morbidity than HS. There are several contributors to donor site morbidity, including paresthesias, anterior knee pain, incision size, and scar cosmesis. Hypoesthesias from nerve injuries are a well-documented consequence of autograft harvest during ACLR that have been shown to correlate with patient dissatisfaction and donor site complaints [37,38]. In support of this, Mouarbes et al. found that HS harvesting was associated with greater hypoesthesias than QT harvest. [13]. While the terminal branch of the intermediate femoral cutaneous nerve does appear to be at risk during QT harvest [38], current evidence seems to suggest that this leads to lesser hypoesthesias than injury to the infrapatellar branch of the saphenous nerve during HS harvest.

While the results of this study support the use of QT in ACLR, there are several technical aspects related to the use of QT autografts that are still not fully understood. These include whether or not to include a patellar bone block versus a soft tissue only graft, full versus partial-thickness grafts, and the optimal method of graft fixation. Kanakamedala et al. 
performed a systematic review evaluating QT graft thickness in ACLR found no difference in functional or clinical outcomes or graft re-rupture rates between partial and full-thickness grafts. [39]. The authors suggested that, given the risks of violation of the joint capsule and increased donor site morbidity with full-thickness grafts, partial-thickness grafts might be preferred; however, surgeons could be justified in using either. Another systematic review looking at graft fixation found no difference in graft re-rupture rate or clinical outcomes between aperture versus suspensory fixation of QT. [40]. None of the aforementioned reviews compared all soft-tissue QT to QT grafts, including a bone plug, and further studies will be valuable in this area. Theoretical benefits to all-soft tissue QT include mitigated risk of patellar fracture and reduced donor site morbidity, although these are rarely reported, and can be minimized by harvesting less than $30 \%$ of the patella [41]. The disadvantages include the lack of bone-to-bone graft healing, which may increase the risk of post-operative graft failure.

\section{Limitations}

There were several limitations to this study, including the quantity and quality of studies included in this review. The majority of the studies were retrospective, had low sample sizes, and were heterogeneous in their reported outcomes, which limits the strength of our conclusions. As mentioned before, there was heterogeneity in graft harvest and preparation between studies. The statistical power in some of the reported outcome measures may also be underpowered to detect a significant difference. While the graft rupture rate for QT might seem unusually low, it is important to note that the mean follow-up time for patients in this review was 27.4 months, so no conclusions can be made regarding the long-term graft re-rupture rate for QT. Additionally, the mean age in this study was 27.9, which represents a slightly older cohort for those undergoing ACLR; however, several studies evaluated QT versus HS in younger patients. Some of the analysed studies did not record graft failure and excluded such patients from their study. In the same vein, only eight studies included clinical outcomes. This introduces a degree of heterogeneity as these failing patients were not always tracked for outcomes. As such, their scores are not reflected, particularly in the hamstring group, as is shown to have an increased tendency to failure and may contribute to a falsely elevated score in this group and lack of statistical significance. Finally, there was no registered protocol done prior to the conduction of this study.

\section{Conclusion}

Our study showed that QT has a lower re-rupture rate than HS in ACLR, with lower donor site morbidity. QT appeared to be slightly better for residual pivot shift, but there was no difference in patient-reported outcomes.

\section{Conflicts of interest}

There is no conflict of interest.

\section{References}

[1] Stanish W, Kirkpatrick J, Rubinovich R. Reconstruction of the anterior cruciate ligament with a quadricep patellar tendon graft. Preliminary results. Can J Appl Sport Sci 1984;9(1):21-4. pmid: 6705125.

[2] Noyes F, Butler D, Grood E, Zernicke R, Hefzy M. Biomechanical analysis of human ligament grafts used in knee-ligament. J Bone Joint Surg Am 1984;66:344-52. pmid: 6699049.

[3] Harris NL, Smith DA, Lamoreaux L, Purnell M. Central quadriceps tendon for anterior cruciate ligament reconstruction: Part I: morphometric and biomechanical evaluation. Am J Sports Med 1997;25(1):23-8. https://doi.org/10.1177/ 036354659702500105.

[4] Stäubli H-U. The quadriceps tendon-patellar bone construct for ACL reconstruction. Knieinstabilität und Knorpelschaden. Springer; 1997. p. 126-39.
[5] Lubowitz JH. Editorial commentary: quadriceps tendon autograft use for anterior cruciate ligament reconstruction predicted to increase. Arthroscopy 2016;32(1):76-7. https://doi.org/10.1016/j.arthro.2015.11.004.

[6] Xerogeanes JW. Quadriceps tendon graft for anterior cruciate ligament reconstruction: the graft of the future. Arthroscopy 2019;35(3):696-7. https:// doi.org/10.1016/j.arthro.2019.01.011.

[7] Sheean AJ, Musahl V, Slone HS, et al. Quadriceps tendon autograft for arthroscopic knee ligament reconstruction: use it now, use it often. Br J Sports Med 2018;52(11): 698-701. https://doi.org/10.1136/bjsports-2017-098769.

[8] Belk JW, Kraeutler MJ, Marshall HA, Goodrich JA, McCarty EC. Quadriceps tendon autograft for primary anterior cruciate ligament reconstruction: a systematic review of comparative studies with minimum 2-year follow-up. Arthrosc J Arthrosc Relat Surg 2018;34(5):1699-707. https://doi.org/10.1016/j.arthro.2018.01.047.

[9] Hurley ET, Calvo-Gurry M, Withers D, Farrington SK, Moran R, Moran CJ. Quadriceps tendon autograft in anterior cruciate ligament reconstruction: a systematic review. Arthrosc J Arthrosc Relat Surg 2018;34(5):1690-8. https:// doi.org/10.1016/j.arthro.2018.01.046.

[10] Ajrawat P, Dwyer T, Whelan D, et al. A comparison of quadriceps tendon autograft with bone-patellar tendon-bone autograft and hamstring tendon autograft for primary anterior cruciate ligament reconstruction: a systematic review and quantitative synthesis. Clin J Sport Med : official journal of the Canadian Academy of Sport Medicine 2019. https://doi.org/10.1097/jsm.0000000000000765.

[11] Mouarbes D, Menetrey J, Marot V, Courtot L, Berard E, Cavaignac E. Anterior cruciate ligament reconstruction: a systematic review and meta-analysis of outcomes for quadriceps tendon autograft versus bone-patellar tendon-bone and hamstring-tendon autografts. Am J Sports Med 2019;47(14):3531-40. https:// doi.org/10.1177/0363546518825340.

[12] Nyland J, Collis P, Huffstutler A, et al. Quadriceps tendon autograft ACL reconstruction has less pivot shift laxity and lower failure rates than hamstring tendon autografts. Knee Surg Sports Traumatol Arthrosc 2020;28(2):509-18. https://doi.org/10.1007/s00167-019-05720-y.

[13] Mouarbes D, Dagneaux L, Olivier M, et al. Lower donor-site morbidity using QT autografts for ACL reconstruction. Knee Surg Sports Traumatol Arthrosc 2020:1-9. https://doi.org/10.1007/s00167-020-05873-1.

[14] Akoto R, Albers M, Balke M, Bouillon B, Hoher J. ACL reconstruction with quadriceps tendon graft and press-fit fixation versus quadruple hamstring graft and interference screw fixation - a matched pair analysis after one year follow up. BMC Muscoskel Disord 2019;20(1):109. https://doi.org/10.1186/s12891-019-2499-y [published Online First: 2019/03/16].

[15] Barie A, Ehmann Y, Jaber A, Huber J, Streich NA. Revision ACL reconstruction using quadriceps or hamstring autografts leads to similar results after 4 years: good objective stability but low rate of return to pre-injury sport level. Knee Surg Sports Traumatol Arthrosc 2019;27(11):3527-35. https://doi.org/10.1007/s00167-01905444-z [published Online First: 2019/03/02].

[16] Cavaignac E, Coulin B, Tscholl P, Nik Mohd Fatmy N, Duthon V, Menetrey J. Is quadriceps tendon autograft a better choice than hamstring autograft for anterior cruciate ligament reconstruction? A comparative study with a mean follow-up of 3.6 years. Am J Sports Med 2017;45(6):1326-32. https://doi.org/10.1177/ 0363546516688665 [published Online First: 2017/03/09].

[17] Haner M, Bierke S, Petersen W. Anterior cruciate ligament revision surgery: ipsilateral quadriceps versus contralateral semitendinosus-gracilis autografts. Arthroscopy 2016;32(11):2308-17. https://doi.org/10.1016/j.arthro.2016.03.020 [published Online First: 2016/05/23].

[18] Hart R, Kucera B, Safi A. [Hamstring versus quadriceps tendon graft in doublebundle anterior cruciate ligament reconstruction]. Acta Chir Orthop Traumatol Cech 2010;77(4):296-303 [published Online First: 2010/11/10], pmid: 21059327.

[19] Lee JK, Lee S, Lee MC. Outcomes of anatomic anterior cruciate ligament reconstruction: bone-quadriceps tendon graft versus double-bundle hamstring tendon graft. Am J Sports Med 2016;44(9):2323-9. https://doi.org/10.1177/ 0363546516650666 [published Online First: 2016/07/02].

[20] Kim SJ, Lee SK, Choi CH, Kim SH, Kim SH, Jung M. Graft selection in anterior cruciate ligament reconstruction for smoking patients. Am J Sports Med 2014; 42(1):166-72. https://doi.org/10.1177/0363546513505191 [published Online First: $2013 / 10 / 12]$.

[21] Lind M, Nielsen TG, Soerensen OG, Mygind-Klavsen B, Fauno P. Quadriceps tendon grafts does not cause patients to have inferior subjective outcome after anterior cruciate ligament (ACL) reconstruction than do hamstring grafts: a 2-year prospective randomised controlled trial. Br J Sports Med 2020;54(3):183-7. https://doi.org/10.1136/bjsports-2019-101000 [published Online First: 2019/11/ $11]$.

[22] Martin-Alguacil JL, Arroyo-Morales M, Martin-Gomez JL, et al. Strength recovery after anterior cruciate ligament reconstruction with quadriceps tendon versus hamstring tendon autografts in soccer players: a randomized controlled trial. Knee 2018;25(4):704-14. https://doi.org/10.1016/j.knee.2018.03.011 [published Online First: 2018/05/20].

[23] Pennock AT, Johnson KP, Turk RD, et al. Transphyseal anterior cruciate ligament reconstruction in the skeletally immature: quadriceps tendon autograft versus hamstring tendon autograft. Orthop J Sports Med 2019;7(9). https://doi.org/ 10.1177/2325967119872450. 2325967119872450 [published Online First: 2019/ 09/27].

[24] Runer A, Wierer G, Herbst E, et al. There is no difference between quadriceps- and hamstring tendon autografts in primary anterior cruciate ligament reconstruction: a 2-year patient-reported outcome study. Knee Surg Sports Traumatol Arthrosc 2018; 26(2):605-14. https://doi.org/10.1007/s00167-017-4554-2 [published Online First: $2017 / 05 / 10]$. 
[25] Sofu H, Sahin V, Gursu S, Yildirim T, Issin A, Ordueri M. Use of quadriceps tendon versus hamstring tendon autograft for arthroscopic anterior cruciate ligament reconstruction: a comparative analysis of clinical results. Eklem Hastalik Cerrahis 2013;24(3):139-43. https://doi.org/10.5606/ehc.2013.31 [published Online First: 2013/11/07].

[26] Todor A, Nistor DV, Caterev S. Clinical outcomes after ACL reconstruction with free quadriceps tendon autograft versus hamstring tendons autograft. A retrospective study with a minimal follow-up two years. Acta Orthop Traumatol Turcica 2019; 53(3):180-3. https://doi.org/10.1016/j.aott.2019.03.004 [published Online First: 2019/03/25].

[27] Vilchez-Cavazos F, Davila-Martinez A, Garza-Castro S, et al. Anterior cruciate ligament injuries treated with quadriceps tendon autograft versus hamstring autograft: a randomized controlled trial. Cir Cir 2020;88(1):76-81. https://doi.org/ 10.24875/CIRU.19001001 [published Online First: 2020/01/23].

[28] Gulotta LV, Kovacevic D, Ying L, Ehteshami JR, Montgomery S, Rodeo SA. Augmentation of tendon-to-bone healing with a magnesium-based bone adhesive. Am J Sports Med 2008;36(7):1290-7. https://doi.org/10.1177/ 0363546508314396.

[29] Petersen W, Laprell H. Insertion of autologous tendon grafts to the bone: a histological and immunohistochemical study of hamstring and patellar tendon grafts. Knee Surg Sports Traumatol Arthrosc 2000;8(1):26-31. https://doi.org/ $10.1007 / \mathrm{s} 001670050006$.

[30] Sasaki N, Farraro KF, Kim KE, Woo SL. Biomechanical evaluation of the quadriceps tendon autograft for anterior cruciate ligament reconstruction: a cadaveric study. Am J Sports Med 2014;42(3):723-30. https://doi.org/10.1177/ 0363546513516603 [published Online First: 2014/01/10].

[31] Stäubli HU, Schatzmann L, Brunner P, Rincón L, Nolte L-P. Mechanical tensile properties of the quadriceps tendon and patellar ligament in young adults. Am J Sports Med 1999;27(1):27-34. https://doi.org/10.1177/03635465990270011301.

[32] Shani RH, Umpierez E, Nasert M, Hiza EA, Xerogeanes J. Biomechanical comparison of quadriceps and patellar tendon grafts in anterior cruciate ligament reconstruction. Arthrosc J Arthrosc Relat Surg 2016;32(1):71-5. https://doi.org/ 10.1016/j.arthro.2015.06.051.
[33] Kyritsis P, Bahr R, Landreau P, Miladi R, Witvrouw E. Likelihood of ACL graft rupture: not meeting six clinical discharge criteria before return to sport is associated with a four times greater risk of rupture. Br J Sports Med 2016;50(15): 946. https://doi.org/10.1136/bjsports-2015-095908.

[34] Hamner DL, Brown CH, Steiner ME, Hecker AT, Hayes WC. Hamstring tendon grafts for reconstruction of the anterior cruciate ligament: biomechanical evaluation of the use of multiple strands and tensioning techniques. JBJS 1999;81(4):549-57. https://doi.org/10.2106/00004623-199904000-00013.

[35] Pujol N, Queinnec S, Boisrenoult P, Maqdes A, Beaufils P. Anatomy of the anterior cruciate ligament related to hamstring tendon grafts. A cadaveric study. Knee 2013; 20(6):511-4. https://doi.org/10.1016/j.knee.2012.10.006.

[36] Wilson TW, Zafuta MP, Zobitz M. A biomechanical analysis of matched bonepatellar tendon-bone and double-looped semitendinosus and gracilis tendon grafts. Am J Sports Med 1999;27(2):202-7.

[37] Sabat D, Kumar V. Nerve injury during hamstring graft harvest: a prospective comparative study of three different incisions. Knee Surg Sports Traumatol Arthrosc 2013;21(9):2089-95. https://doi.org/10.1007/s00167-012-2243-8.

[38] Horner G, Dellon AL. Innervation of the human knee joint and implications for surgery. Clin Orthop Relat Res 1994;(301):221-6. pmid: 8156678.

[39] Kanakamedala AC, Obioha OA, Arakgi ME, Schmidt PB, Lesniak BP, Musahl V. No difference between full thickness and partial thickness quadriceps tendon autografts in anterior cruciate ligament reconstruction: a systematic review. Knee Surg Sports Traumatol Arthrosc 2019;27(1):105-16. https://doi.org/10.1007/s00167-0185042-z.

[40] Crum RJ, de Sa D, Kanakamedala AC, Obioha OA, Lesniak BP, Musahl V. Aperture and suspensory fixation equally efficacious for quadriceps tendon graft fixation in primary ACL reconstruction: a systematic review. J Knee Surg 2019. https:// doi.org/10.1055/s-0039-1685160 [published Online First: 2019/04/09].

[41] Ferrer GA, Miller RM, Murawski CD, et al. Quantitative analysis of the patella following the harvest of a quadriceps tendon autograft with a bone block. Knee Surg Sports Traumatol Arthrosc 2016;24(9):2899-905. https://doi.org/10.1007/ s00167-015-3550-7 [published Online First: 2015/03/10]. 